International Electronic Journal of Algebra

Volume 26 (2019) 13-28

DOI: $10.24330 /$ ieja. 586882

\title{
EXTENDING TABLE ALGEBRAS TO HOPF ALGEBRAS
}

\author{
Allen Herman and Gurmail Singh \\ Received: 10 May 2018; Revised: 6 February 2019; Accepted: 13 February 2019 \\ Communicated by Abdullah Harmancı
}

\begin{abstract}
Let $A$ be a table algebra with standard basis $\mathbf{B}$, multiplication $\mu$, unit map $\eta$, skew-linear involution $*$, and degree map $\delta$. In this article we study the possible coalgebra structures $(A, \Delta, \delta)$ on $A$ for which $(A, \mu, \eta, \Delta, \delta)$ becomes a Hopf algebra with respect to some antipode. We show that such Hopf algebra structures are not always available for noncommutative table algebras. On the other hand, commutative table algebras will always have a Hopf algebra structure induced from an algebra-isomorphic group algebra. To illustrate our approach, we derive Hopf algebra comultiplications on table algebras of dimension 2 and 3 .
\end{abstract}

Mathematics Subject Classification (2010): 16T30, 05E30

Keywords: Table algebra, Hopf algebra, fusion ring, association scheme

\section{Introduction}

A bialgebra $(A, \mu, \eta, \Delta, \delta)$ is both an associative algebra and a coassociative coalgebra $A$ over a commutative ring $K$ with multiplication $\mu: A \otimes A \rightarrow A$, unit map $\eta: K \rightarrow A$, comultiplication $\Delta: A \rightarrow A \otimes A$, and counit $\delta: A \rightarrow K$, that satisfies extra compatibility conditions that $\Delta: A \rightarrow A \otimes A$ and $\delta$ are algebra homomorphisms and $\delta \circ \eta=i d_{K}$. For non-experts, the algebra condition on $(A, \mu, \eta)$ is

$$
\mu \circ(i d \otimes \eta)=\mu \circ(\eta \otimes i d)=i d
$$

as maps on $A \otimes K \simeq K \otimes A \simeq A$, and the coalgebra condition on $(A, \Delta, \delta)$ is obtained by reversing arrows:

$$
(i d \otimes \delta) \circ \Delta=(\delta \otimes i d) \circ \Delta=i d .
$$

An algebra anti-automorphism $S: A \rightarrow A$ of a bialgebra $(A, \mu, \eta, \Delta, \delta)$ is called an antipode when

$$
\eta \circ \delta=\mu \circ(\mathcal{S} \otimes i d) \circ \Delta=\mu \circ(i d \otimes \mathcal{S}) \circ \Delta .
$$

The first author has been supported by an NSERC Discovery Grant. 
A Hopf algebra is a bialgebra with antipode. Hopf algebras are a class of bialgebras that includes group algebras, since it is well-known that a group algebra $K G$ of a group $G$ over $K$ becomes a Hopf algebra over $K$ when we define comultiplication by $\Delta(g)=g \otimes g$ and counit by $\delta(g)=1$, and the antipode by $\mathcal{S}(g)=g^{-1}$, for all $g \in G$.

In the case where the base field is the field of complex numbers $\mathbb{C}$ (which we will assume from now on), table algebras are associative algebras that also generalize group algebras. (For an extensive history on the evolution and scope of this and similar notions, we refer the reader to Blau's survey article [1].) A table algebra of finite rank $r$ is an $r$-dimensional associative algebra $A$ with skew-linear involution * whose distinguished basis $\mathbf{B}=\left\{1=b_{0}, b_{1}, \ldots, b_{r-1}\right\}$ satisfies the following three properties:

TA1: The structure constants relative to $\mathbf{B}$ are nonnegative real numbers. We denote these by $\lambda_{i j k}$ for $i, j, k \in\{0,1, \ldots, r-1\}$, where $b_{i} b_{j}=\sum_{k} \lambda_{i j k} b_{k}$, for all $b_{i}, b_{j} \in \mathbf{B}$.

TA2: $\mathbf{B}^{*}=\mathbf{B}$; in particular, $b_{0}^{*}=b_{0}=1$ is the multiplicative identity of the algebra, and for all $b_{i} \in \mathbf{B}$ there is a unique $i^{*} \in\{0,1, \ldots, r-1\}$ such that $\left(b_{i}\right)^{*}=b_{i^{*}}$.

TA3: For all $i \in\{0,1, \ldots, r-1\}, b_{i^{*}}$ is the unique pseudo-inverse of $b_{i}$; this means for all $i, j \in\{0,1, \ldots, r-1\}, \lambda_{i j 0}>0 \Longleftrightarrow j=i^{*}$, and for all $i \in\{0,1, \ldots, r-1\}$, $\lambda_{i i^{*} 0}=\lambda_{i^{*} i 0}$.

The complex group algebra $\mathbb{C} G$ of a finite group $G$ is a table algebra with distinguished basis $G$. Its skew-linear involution is given by $(\alpha g)^{*}=\bar{\alpha} g^{-1}$, for all $g \in G$, $\alpha \in \mathbb{C}$, and the pseudo-inverse of $g \in G$ is its group inverse $g^{-1}$. The adjacency algebras of association schemes are another important example of table algebras, in which the roles of involution and pseudo-inverse are played by conjugate transpose and transpose, respectively. Note that table algebras, being finite-dimensional algebras with involution over $\mathbb{C}$, are always semisimple algebras. For further overview of the theory of table algebras, see Blau's survey [1].

As is customary we will write $b_{0}$ for the multiplicative identity of a table algebra, so the unit map of a table algebra is given by $\eta(\alpha)=\alpha b_{0}$, for $\alpha \in \mathbb{C}$, and multiplication $\mu$ is as defined by property TA1. Since table algebras are a class of associative algebras that includes group algebras, and group algebras become Hopf algebras in a natural way, it makes sense to ask:

Is there a way to define a counit $\delta$, comultiplication $\Delta$, and antipode $\mathcal{S}$ on a table algebra $(A, \mu, \eta)$ so that $(A, \mu, \eta, \Delta, \delta)$ becomes a Hopf algebra with antipode $\mathcal{S}$ ? 
The main results of this article show that the answer to this question is NO for some noncommutative table algebras, but YES for all commutative table algebras. Since fusion rule algebras can be characterized as commutative table algebras after a basis renormalization (see [1]), the answer is also YES for fusion rule algebras. The negative answer is a consequence of the existence of noncommutative table algebras of dimensions 5 and 7, combined with the fact that Hopf algebras of prime dimension must be isomorphic to group algebras. Since commutative table algebras of dimension $r$ are algebra isomorphic to the group algebra of any abelian group of order $r$, for each distinct isomorphism type of these groups there will be a distinct Hopf algebra structure on the table algebra. To Hopf algebraists, the possible comultiplications on commutative semisimple algebras are well-known to be the coalgebra structures for duals of group algebras. We offer a self-contained proof of this fact that also produces the values of the comultiplication on the basis of primitive idempotents. By interpolating between the character tables of the table algebra and the group we can produce formulas for the values of the Hopf algebra comultiplication map on elements of $\mathbf{B}$ for the most natural choices of counits. We will apply this approach to give explicit Hopf algebra comultiplications for table algebras in dimensions 2 and 3.

\section{Choosing the counit and antipode of a table algebra}

Let $A$ be a table algebra with distinguished basis B. Since each distinguished basis element $b_{i}$ can be realized as a nonnegative matrix in its regular representation, there is a unique algebra homomorphism $\delta: \mathbb{C B} \rightarrow \mathbb{C}$ called the degree map for which $\delta\left(b_{i}\right)>0$ for all $b_{i} \in \mathbf{B}$ [1]. Since $\delta$ is an algebra homomorphism, it is compatible with $\mu$, and $\delta\left(b_{0}\right)=1$ implies $\delta(\eta(z))=z$, for all $z \in \mathbb{C}$, so it is also compatible with our unit map. In the case $\mathbf{B}$ is a finite group the degree map is the augmentation map, which is the counit for the usual Hopf algebra structure on the group algebra. So the degree map of the table algebra is a good candidate for the counit.

In choosing the degree map for the counit, we can scale the distinguished basis elements by positive constants to arrange that our renormalized basis satisfies $\delta\left(b_{i}\right)=\lambda_{i i^{*} 0}$, for all $b_{i} \in \mathbf{B}$ (see [1]). We call such a table algebra basis standard, and from now on assume our table algebra bases to be standard ones.

When $A$ is a table algebra, it has a natural involution $*$ that is skew-linear relative to its defining basis $\mathbf{B}$. This means the conjugate involution $\bar{*}$ given by $\left(\alpha b_{i}\right)^{\bar{*}}=\overline{\bar{\alpha}} b_{i^{*}}=\alpha b_{i^{*}}$ for all $\alpha \in \mathbb{C}$ and $b_{i} \in \mathbf{B}$ will be linear relative to the basis $\mathbf{B}$ 
of $A$. In the case of group algebras $\bar{*}$ corresponds to the linear map that restricts to inversion on the group basis, which gives the antipode for the Hopf algebra structure on the group algebra. So $\bar{*}$ is one candidate for our antipode. However, the next example shows it will not always be the correct one.

Remark 2.1. For group algebras $\mathbb{C} G$ of finite groups $G$, it is in fact the case that any representation of degree one can be the counit of a Hopf algebra structure on $\mathbb{C} G$. When $\chi \in \operatorname{Irr}(G)$ is a nontrivial representation $\chi: G \rightarrow \mathbb{C}^{\times}$, then it is straightforward to show that the set $G_{\chi}=\left\{g_{\chi}:=\chi(g)^{-1} g: g \in G\right\}$ is a group, isomorphic to $G$, that will be another group basis of $\mathbb{C} G$. As a representation of the algebra $\mathbb{C} G, \chi$ restricts to the trivial character of $G_{\chi}$. For the antipode of $\mathbb{C} G_{\chi}$ we need to consider a different involution $\bar{*}^{\prime}$ of $\mathbb{C} G$, which is given by $\left(\alpha g_{\chi}\right)^{\bar{F}^{\prime}}=\left(\bar{\alpha} g_{\chi}\right)^{\bar{*}}=\alpha g_{\chi}^{-1}$, for all $\alpha \in \mathbb{C}$ and $g_{\chi} \in G_{\chi}$. So we can define a new Hopf algebra comultiplication on $\mathbb{C} G$ by setting $\Delta_{\chi}\left(g_{\chi}\right)=g_{\chi} \otimes g_{\chi}$, and this Hopf algebra structure on $\mathbb{C} G$ has counit $\chi$ and antipode $\bar{*}^{\prime}$.

It remains to find good candidates for the comultiplication map $\Delta$ that will make $(A, \mu, \eta, \Delta, \delta)$ into a Hopf algebra with respect to some suitable antipode. There is, however, no obvious candidate for $\Delta$. One table algebra comultiplication given by $\Delta\left(b_{i}\right)=\frac{1}{\delta\left(b_{i}\right)}\left(b_{i} \otimes b_{i}\right)$, for all $b_{i} \in \mathbf{B}$ was considered earlier by Hanaki in [2] in the case where the table algebra arises from an association scheme. He observed it does not produce a Hopf algebra. While this $\Delta$ is coassociative, it is not an algebra homomorphism, so it will not produce a bialgebra structure. Hanaki's motivation for considering bialgebra structures that extend the algebra structure of a table algebra is that the tensor product of two representations of $A$ becomes a representation of $A$ on pre-composing with algebra homomorphism $\Delta$. So even if we cannot find a Hopf algebra structure extending the table algebra structure, it would still be useful to find a bialgebra structure.

\section{Cases of non-existence}

Let $A$ be a table algebra with standard basis $\mathbf{B}$ and degree map $\delta$. A simple strategy to find a comultiplication $\Delta$ that makes $(A, \mu, \eta, \Delta, \delta)$ into a bialgebra is to produce an algebra isomorphism of $A$ with a known bialgebra $H$, and borrow the necessary coalgebra structure from $H$. If, in addition, this $H$ is a Hopf algebra, then its antipode will also induce one on $A$. Since our table algebras are semisimple algebras, we can focus on finite-dimensional Hopf algebras that are semisimple as algebras. These are known to be isomorphic as Hopf algebras to group algebras or their duals in the following cases: 
(i) $H$ is commutative (as this implies it is cocommutative and cosemisimple) [6] (see also the general prime and prime-square dimension cases treated in [7] and [4]);

(ii) $H$ has dimension divisible by two distinct primes [5].

In the next section we will show that when table algebras are algebra isomorphic to group algebras, our table algebra will have a Hopf algebra structure whose counit is our degree map $\delta$.

On the other hand, we know from results of Masuoka in [4] and [5] that the smallest dimension for which there are noncommutative semisimple Hopf algebras not isomorphic to group algebras is 8 (see [6, pg. 528-529]). Since noncommutative table algebras of dimension 5 and 7 have been shown to exist in [3], we can conclude these table algebras cannot possess a Hopf algebra structure at all.

Theorem 3.1. Noncommutative table algebras exist for which there is no possible Hopf algebra comultiplication.

Remark 3.2. Noncommutative table algebras of dimension 5 are not realized as the adjacency algebras of association schemes, but there are noncommutative table algebras of dimension 7 that are realized as adjacency algebras of association schemes, the smallest of which occurs for an association scheme of order 12 .

Remark 3.3. The results of [3] are given in the slightly more general setting of reality-based algebras with a positive degree map. These are semisimple algebras with a distinguished basis satisfying most of the table algebra axioms (TA1)-(TA3) except the real strucuture constants $\lambda_{i j k}$ are allowed to be negative when $k \neq 0$ or $j \neq i^{*}$, and the positive degree map is an extra assumption. All of the results of this article apply immediately in this more general setting. In particular our results below for commutative table algebras generalize immediately to commutative reality based algebras with positive degree map, which are commonly known as $C$-algebras.

\section{Hopf algebra structures induced by group algebra isomorphism}

Theorem 4.1. Let $A$ be an $r$-dimensional table algebra with degree map $\delta$. If $A$ is algebra isomorphic to a group algebra $\mathbb{C} G$ for a finite group $G$ of order $r$, then the algebra structure of $A$ can be extended to a Hopf algebra structure on $A$ whose counit is $\delta$.

Proof. Let $(A, \mu, \eta)$ be the algebra structure on $A$. Suppose $\Theta: A \rightarrow \mathbb{C} G$ is an algebra isomorphism. Then $\chi:=\delta \circ \Theta^{-1}$ is a degree one representation, so as in Remark 2.1 we can adjust the group basis of $\mathbb{C} G$ if necessary to arrange that 
coalgebra structure on $\mathbb{C} G$ has comultiplication given by $\Delta_{G}(g)=g \otimes g$, for all $g \in G$, counit $\chi$, and antipode $S(g)=g^{-1}$, for all $g \in G$. We can then see that $A$ has the Hopf algebra structure whose bialgebra is $\left(A, \mu, \eta,\left(\Theta^{-1} \otimes \Theta^{-1}\right) \circ \Delta \circ \Theta, \delta\right)$ and whose antipode is $\Theta^{-1} \circ S \circ \Theta$.

Remark 4.2. In the case of commutative table algebras of dimension $r$, the calculation of $\Delta$ in Theorem 4.1 can done by interpolating between the character tables of the table algebra and the group.

Let $N$ be the character table of the abelian group $G$ and let $E=\left\{e_{\chi}: \chi \in\right.$ $\operatorname{Irr}(G)\}$ be the basis of primitive idempotents for $\mathbb{C} G$. Then the formula $g=$ $\sum_{\chi} \chi(g) e_{\chi}$ for all $g \in G$ tells us that $N$ is the change of basis matrix from the basis $E$ to the basis $G$.

On the other hand, if $P$ is the character table (first eigenmatrix) of the commutative table algebra $A$ with respect to the basis $\mathbf{B}$, then the primitive idempotent formula

$$
e_{\chi}=\frac{m_{\chi}}{n} \sum_{i} \frac{\chi\left(b_{i}^{*}\right)}{\delta\left(b_{i}\right)} b_{i}
$$

indicates that the change of basis matrix from $E$ to the standard basis $\mathbf{B}$ is the matrix $M$ obtained by dividing each column of $P$ by the degree of the corresponding element $b_{i}$ and multiplying each row of $P$ by the multiplicity of the corresponding character $\chi \in \operatorname{Irr}(A)$. The composition of these change of basis matrices realizes a $*$-algebra isomorphism between $A$ and $\mathbb{C} G$ because the elements of $E$ are *invariant; i.e. $e_{\chi}^{*}=e_{\chi}$ for all $e_{\chi} \in E$ [1, Proposition 2.9]. Furthermore, the degree map and augmentation map both correspond to the first rows of $P$ and $N$, so they are identified and so the counit is preserved. Using the change of basis matrix $M N^{-1}$ allows us to write elements of $\mathbf{B}$ in terms of the basis $G$, then calculate the value of the comultiplication using $\Delta_{G}$, and the inverse change of basis $N M^{-1}$ allows us to write the elements of $G$ in terms of the basis B. So this gives an explicit formula for a Hopf algebra comultiplication that comes directly from the entries of $M N^{-1}$ and $N M^{-1}$. This technique will be used in the next section to generate explicit Hopf algebra comultiplication formulas for table algebras of small rank.

\section{Values of Hopf algebra comultiplications on primitive idempotents}

Experts familiar with the theory of finite-dimensional Hopf algebras are aware that finite-dimensional commutative semisimple Hopf algebras are cocommutative and cosemisimple, and thus isomorphic to group algebras or their duals. These results are normally established using properties of integrals and the convolution algebra. In this section we give a self-contained proof of this that has the side-benefit 
of producing a useful formula for the values of the Hopf algebra comultiplication on the basis of primitive idempotents. The proof given here is due to the referee of an earlier version of this article, it has the benefit of showing $S^{2}=1$.

Theorem 5.1. Let $A$ be a commutative semisimple algebra over $\mathbb{C}$, and let $E$ be its basis of primitive idempotents $\left\{e_{\chi}: \chi \in \operatorname{Irr}(A)\right\}$. Suppose the algebra structure on $A$ extends to a Hopf algebra structure $(A, \mu, \eta, \Delta, \delta)$ with antipode $S$. Then there is an abelian group structure on $\operatorname{Irr}(A)$ such that for all $e_{\chi}$ in $E$,

$$
\Delta\left(e_{\chi}\right)=\sum_{\psi \star \theta=\chi} e_{\psi} \otimes e_{\theta}
$$

Proof. Since the antipode $S$ is an anti-automorphism of $A, S$ acts as a permutation on the set $E$. Let $S\left(e_{\chi}\right)=e_{S(\chi)}$ for all $\chi \in \operatorname{Irr}(A)$. Also, $\Delta: A \rightarrow A \otimes A$ is an algebra homomorphism, so $\Delta\left(e_{\chi}\right)$ is an idempotent of $A \otimes A$. Since $A$ is a finite-dimensional commutative semisimple algebra over an algebraically closed field, there is a subset $C_{\chi}$ of $\operatorname{Irr}(A) \times \operatorname{Irr}(A)$ (possibly empty) such that

$$
\Delta\left(e_{\chi}\right)=\sum_{(\theta, \phi) \in C_{\chi}}\left(e_{\theta} \otimes e_{\phi}\right)
$$

Now,

$$
1 \otimes 1=\Delta(1)=\sum_{\chi \in \operatorname{Irr}(A)} \Delta\left(e_{\chi}\right)=\sum_{\chi \in \operatorname{Irr}(A)} \sum_{(\theta, \phi) \in C_{\chi}}\left(e_{\theta} \otimes e_{\phi}\right)
$$

so every $(\theta, \phi) \in \operatorname{Irr}(A) \times \operatorname{Irr}(A)$ is an element of $C_{\chi}$ for some $\chi \in \operatorname{Irr}(A)$. Since $1 \otimes 1=\sum_{\theta \in \operatorname{Irr}(A)} \sum_{\phi \in \operatorname{Irr}(A)}\left(e_{\theta} \otimes e_{\phi}\right)$, it follows that for every $\theta, \phi \in \operatorname{Irr}(A)$, there is a unique $\chi \in \operatorname{Irr}(A)$ such that $(\theta, \phi) \in C_{\chi}$. Therefore, setting $\theta \star \phi=\chi$ iff $(\theta, \phi) \in C_{\chi}$, for all $\theta, \phi \in \operatorname{Irr}(A)$ gives a well-defined binary operation on $\operatorname{Irr}(A)$.

We have that the counit $\delta \in \operatorname{Irr}(A)$ satisfies the condition that $(1 \otimes \delta) \circ \Delta$ is equal to the natural isomorphism $(1 \otimes 1)^{-1}: A \rightarrow A \otimes \mathbb{C}$. So whenever $\chi \star \delta=\psi$, we have

$$
e_{\psi} \otimes 1=(1 \otimes \delta) \Delta\left(e_{\psi}\right)=\sum_{(\theta, \phi) \in C_{\psi}}\left(e_{\theta} \otimes \delta\left(e_{\phi}\right)\right)=\sum_{\theta:(\theta, \delta) \in C_{\psi}}\left(e_{\theta} \otimes 1\right) .
$$

This implies that $(\theta, \delta) \in C_{\psi}$ iff $\theta=\psi$. Since $(\chi, \delta) \in C_{\psi}$ we also have $\chi=\psi$. Therefore, for each $\chi \in \operatorname{Irr}(A)$ we have $\chi \star \delta=\chi$. Similarly we can show $\delta \star \chi=\chi$, and so $\delta$ is a $\star$-identity in $\operatorname{Irr}(A)$.

Finally,

$$
\delta_{\chi \delta} 1_{A}=\eta\left(\chi\left(e_{\delta}\right)\right)=\mu(S \otimes 1) \Delta\left(e_{\delta}\right)=\sum_{(\theta, \phi) \in C_{\delta}} e_{S(\theta)} e_{\phi}=\sum_{(\theta, S(\theta)) \in C_{\delta}} e_{S(\theta)},
$$


so $C_{\delta}=\{(\theta, S(\theta)): \theta \in \operatorname{Irr}(A)\}$. Therefore, for all $\theta \in \operatorname{Irr}(A)$, we have $\theta \star S(\theta)=\delta$. So for all $\theta \in \operatorname{Irr}(A), S(\theta)$ is a right inverse for $\theta$, and we can similarly show it will be a left inverse. It follows then that $S^{2}$ is the identity permutation on $\operatorname{Irr}(A)$, and hence $S^{2}$ acts as the identity operation on $A$.

Finally, from coassociativity we have $(1 \otimes \Delta) \circ \Delta=(\Delta \otimes 1) \otimes \Delta$, applied to $e_{\chi}$ for $\chi \in \operatorname{Irr}(A)$ this gives

$$
\sum_{\theta \star \phi=\chi} \sum_{\alpha \star \beta=\theta}\left(e_{\alpha} \otimes e_{\beta} \otimes e_{\phi}\right)=\sum_{\alpha \star \psi=\chi} \sum_{\beta \star \phi=\psi}\left(e_{\alpha} \otimes e_{\beta} \otimes e_{\phi}\right) .
$$

Now, $e_{\alpha} \otimes e_{\beta} \otimes e_{\phi}$ appears on the left when $(\alpha \star \beta) \star \phi=\chi$, and it appears on the right when $\alpha \star(\beta \star \phi)=\chi$. Since this holds for every $\chi \in \operatorname{Irr}(A), \star$ is an associative operation on $\operatorname{Irr}(A)$. Therefore, $\star$ is a group operation.

We now have that the coalgebra structure of $(A, \Delta, \delta)$ is the dual of the group algebra $\mathbb{C}[\operatorname{Irr}(A)]$ for the group $(\operatorname{Irr}(A), \star)$. Since this has the same dimension as $A$, the group $(\operatorname{Irr}(A), \star)$ must be abelian. This proves the theorem.

Remark 5.2. The coalgebra structure on $A=\mathbb{C} E$ is determined up to coalgebra isomorphism by the isomorphism type of the group $(\operatorname{Irr}(A), \star)$. If two of these comultiplications defined on the same basis set $E$ produce isomorphic coalgebra structures, then there would be a permutation $\mathcal{P}$ of $E$ for which

$$
\Delta\left(\mathcal{P}\left(e_{\chi}\right)\right)=(\mathcal{P} \otimes \mathcal{P}) \sum_{\psi \phi=\chi}\left(e_{\psi} \otimes e_{\phi}\right)
$$

for all $\chi \in \hat{G}$. But $\mathcal{P}$ induces a permutation $\sigma$ on $\hat{G}$ satisfying

$$
\Delta\left(e_{\sigma(\chi)}\right)=\sum_{\psi \phi=\chi}\left(e_{\sigma(\psi)} \otimes e_{\sigma(\phi)}\right)
$$

for all $\chi \in \hat{G}$, so the two corresponding group multiplication tables would have to be permutation isomorphic. But this implies the two groups would be isomorphic. So the group algebras of two nonisomorphic abelian groups of order $r$ are never isomorphic as Hopf algebras.

\section{Table algebras of dimensions 2 and 3}

In this section we apply the character table approach indicated in Remark 4.2 to produce explicit formulas for Hopf algebra comultiplications for table algebras of ranks 2 and 3. Since these are prime dimension, these Hopf algebra structures will be unique up to isomorphism.

Example 6.1. Let $\mathbf{B}=\left\{1=b_{0}, b_{1}\right\}$ be the standard basis for a table algebra $A$ of dimension 2. Since the involution must fix the multiplicative identity, it also sends 
$b_{1}$ to $b_{1}$. Let $\delta: A \rightarrow \mathbb{C}$ be the degree map of our table algebra. If $k$ is the positive real number for which $\delta\left(b_{1}\right)=k$, then the assumption $\mathbf{B}$ is a standard basis implies $\delta\left(b_{1}\right)=\lambda_{110}=k$. Since $\delta$ is a $\mathbb{C}$-linear map for which $\delta\left(b_{1}^{2}\right)=\delta\left(b_{1}\right)^{2}$, we must have

$$
\mu\left(b_{1} \otimes b_{1}\right)=b_{1}^{2}=k b_{0}+(k-1) b_{1} .
$$

As $b_{0}$ is the multiplicative identity, this completely determines the multiplication map $\mu$ and the unit map $\eta$ of $A$. Our antipode $\bar{*}$ is the identity map on $A$ in this case, because $*$ fixes $b_{0}$ and hence also $b_{1}$.

To use our approach, we will generate *-algebra isomorphisms between $A$ and $\mathbb{C} C_{2}$ using their character tables. The character table of $\mathbb{C} C_{2}$ relative to the group basis $C_{2}$ is $N=\left[\begin{array}{cc}1 & 1 \\ 1 & -1\end{array}\right]$, and $N$ is precisely the change of basis matrix from the basis of primitive idempotents $E$ to the group basis $C_{2}$.

On the other hand, the character table of the 2-dimensional table algebra determined by the parameter $k$ relative to its standard basis $\mathbf{B}$ is

\begin{tabular}{c|cc|c} 
& $b_{0}$ & $b_{1}$ & $m_{\chi}$ \\
\hline$\delta$ & 1 & $k$ & 1 \\
$\phi$ & 1 & -1 & $k$
\end{tabular}

The last column gives the multiplicity of the irreducible character. To obtain the change of basis matrix that converts from coefficients in the basis of primitive idempotents $E$ of $\mathbb{C B}$ to the table algebra basis $\mathbf{B}$, we divide the columns corresponding to the elements $b_{i} \in \mathbf{B}$ by the degree $\delta\left(b_{i}\right)$, and the row corresponding to each $\chi \in \operatorname{Irr}(A)$ by the multiplicity $m_{\chi}$. So the change of basis matrix form $E$ to $\mathbf{B}$ is

$$
M=\left[\begin{array}{cc}
1 & 1 \\
k & -1
\end{array}\right], \text { and its inverse is } M^{-1}=\frac{1}{k+1}\left[\begin{array}{cc}
1 & 1 \\
k & -1
\end{array}\right] .
$$

The change of basis matrix from the basis $C_{2}$ to $\mathbf{B}$ is thus $M N^{-1}$, and from $\mathbf{B}$ to $C_{2}$ is $N M^{-1}$.

Therefore, the element $g=e_{\delta}-e_{\chi}=\frac{1-k}{k+1} b_{0}+\frac{2}{k+1} b_{1}$ is an element with $g^{2}=b_{0}$ for which $\left\{b_{0}, g\right\} \simeq C_{2}$ is a group basis for $A$. Note that $\delta(g)=1$ and $g^{*}=g$, so the induced Hopf algebra structure on $A$ has counit $\delta$ and antipode $\bar{*}$.

Let $\Delta$ be the Hopf algebra comultiplication on the group algebra $\mathbb{C}\langle g\rangle$ with $\Delta\left(b_{0}\right)=b_{0}$ and $\Delta(g)=g \otimes g$. We wish to compute a formula for $\Delta\left(b_{i}\right)$ in terms of the basis of elementary tensors $b_{i j}:=b_{i} \otimes b_{j}$ for $b_{i}, b_{j} \in \mathbf{B}$. Since our change of 
basis matrices also tell us $b_{1}=\frac{k-1}{2} b_{0}+\frac{k+1}{2} g$, we can compute

$$
\begin{aligned}
\Delta\left(b_{1}\right) & =\frac{k-1}{2} \Delta\left(b_{0}\right)+\frac{k+1}{2} \Delta(g) \\
& =\frac{k-1}{2}\left(b_{0} \otimes b_{0}\right)+\frac{k+1}{2}(g \otimes g) \\
& =\frac{k-1}{2}\left(b_{0} \otimes b_{0}\right)+\frac{k+1}{2}\left[\left(\frac{1-k}{k+1} b_{0}+\frac{2}{k+1} b_{1}\right) \otimes\left(\frac{1-k}{k+1} b_{0}+\frac{2}{k+1} b_{1}\right)\right] \\
& =\left[\frac{k-1}{2}+\frac{(1-k)^{2}}{2(k+1)}\right] b_{00}+\frac{(k+1)(2)(1-k)}{2(k+1)^{2}}\left[b_{01}+b_{10}\right]+\frac{(2)(2)(k+1)}{2(k+1)^{2}} b_{11} \\
\Longrightarrow \Delta\left(b_{1}\right) & =\frac{k(k-1)}{k+1} b_{00}+\frac{1-k}{k+1}\left[b_{01}+b_{10}\right]+\frac{2}{k+1} b_{11} .
\end{aligned}
$$

Example 6.2. The Hopf algebra structure of a triangle. When $k$ is a positive integer larger than 1 , the adjacency algebra of a complete graph on $k+1$ vertices is the table algebra of dimension 2 in Example 6.1 determined by the parameter $k$. If we let $b_{0}$ be the $(k+1) \times(k+1)$ identity matrix and $b_{1}$ be the adjacency matrix of the complete graph on $k+1$ vertices, then the formula in Example 6.1 defines the Hopf algebra structure of a complete graph on $k+1$ vertices. Its comultiplication takes values in the space $(k+1)^{2} \times(k+1)^{2}$-matrices spanned by the $b_{i j}$ 's, where $b_{i j}=b_{i} \otimes b_{j}$. For example, when $b_{1}$ is the adjacency matrix of a triangle (the case $k=2$ ), then the Hopf algebra structure is determined by the $9 \times 9$ matrix

$$
\Delta\left(b_{1}\right)=\frac{1}{3}\left(2 b_{00}-b_{01}-b_{10}+2 b_{11}\right) .
$$

Example 6.3. Nonreal table algebras of dimension 3. Suppose $A$ is a 3 -dimensional nonreal table algebra with standard basis $\mathbf{B}=\left\{b_{0}, b_{1}, b_{2}\right\}$, with $b_{1}^{*}=b_{2}$. The table algebra conditions imply that there is a unique $u \geq 0$ such that $b_{1} b_{2}=$ $(2 u+1) b_{0}+u b_{1}+u b_{2}$, and $b_{1}^{2}=u b_{1}+(u+1) b_{2}$. So $\delta\left(b_{1}\right)=\delta\left(b_{2}\right)=2 u+1$.

For $G=C_{3}$ the character table is

$$
N=\left[\begin{array}{ccc}
1 & 1 & 1 \\
1 & \zeta & \zeta^{2} \\
1 & \zeta^{2} & \zeta
\end{array}\right]
$$

for a primitive cube root of unity $\zeta$. We again view $N$ as the change of basis matrix from the basis of primitive idempotents $E$ to the basis $G$. It is important for our approach that the first row of $N$ corresponds to the trivial character, the ordering of the second and third columns is reversed it results in a permutation of some of our structure constants for the Hopf algebra comultiplication.

The character table of the 3 -dimensional nonreal table algebra determined by the parameter $u$ can be calculated from the eigenvalues of the regular matrices of 
$\left\{b_{0}, b_{1}, b_{2}\right\}:$

\begin{tabular}{c|ccc|c} 
& $b_{0}$ & $b_{1}$ & $b_{1}^{*}$ & $m_{\chi}$ \\
\hline$\delta$ & 1 & $2 u+1$ & $2 u+1$ & 1 \\
$\phi$ & 1 & $\frac{1}{2}(-1+\sqrt{-3-4 u})$ & $\frac{1}{2}(-1-\sqrt{-3-4 u})$ & $2 u+1$ \\
$\bar{\phi}$ & 1 & $\frac{1}{2}(-1-\sqrt{-3-4 u})$ & $\frac{1}{2}(-1+\sqrt{-3-4 u})$ & $2 u+1$
\end{tabular}

To obtain the change of basis matrix $M$ that changes the basis from $E$ to $\mathbf{B}$, we multiply each row by the multiplicity of the corresponding irreducible character and divide each column by the degree of the corresponding element. So

$$
M=\left[\begin{array}{ccc}
1 & 1 & 1 \\
2 u+1 & \frac{1}{2}(-1+\sqrt{-3-4 u}) & \frac{1}{2}(-1-\sqrt{-3-4 u}) \\
2 u+1 & \frac{1}{2}(-1-\sqrt{-3-4 u}) & \frac{1}{2}(-1+\sqrt{-3-4 u})
\end{array}\right] .
$$

Again it is important here that the first row of $M$ corresponds to the degree map. Now we use the change of basis matrices $M N^{-1}$ and $N M^{-1}$ to express $b_{1}=\alpha e+$ $\beta g+\gamma g^{-1}$ and $g=r b_{0}+s b_{1}+t b_{2}$, where $\alpha, \beta, \gamma$ are the entries in the second row of $M N^{-1}$ and $r, s, t$ are the entries in the second row of $N M^{-1}$. From these we can obtain a formula for $\Delta\left(b_{1}\right)$ :

$$
\begin{aligned}
\Delta\left(b_{1}\right)= & \alpha(e \otimes e)+\beta(g \otimes g)+\gamma\left(g^{-1} \otimes g^{-1}\right) \\
= & \alpha b_{00}+\beta\left[\left(r b_{0}+s b_{1}+t b_{2}\right) \otimes\left(r b_{0}+s b_{1}+t b_{2}\right)\right] \\
& \quad+\gamma\left[\left(\bar{r} b_{0}+\bar{t} b_{1}+\bar{s} b_{2}\right) \otimes\left(\bar{r} b_{0}+\bar{t} b_{1}+\bar{s} b_{2}\right)\right] \\
= & \left(\alpha+\beta r^{2}+\gamma \bar{r}^{2}\right) b_{00}+(\beta r s+\gamma \bar{r} \bar{t}) b_{01}+(\beta r t+\gamma \bar{r} \bar{s}) b_{02} \\
& +(\beta s r+\gamma \bar{t} \bar{r}) b_{10}+\left(\beta s^{2}+\gamma \bar{t}^{2}\right) b_{11}+(\beta s t+\gamma \bar{t} \bar{s}) b_{12} \\
& +(\beta t r+\gamma \bar{s} \bar{r}) b_{20}+(\beta t s+\gamma \bar{s} \bar{t}) b_{21}+\left(\beta t^{2}+\gamma \bar{s}^{2}\right) b_{22} .
\end{aligned}
$$

Our formula for $\Delta\left(b_{1}\right)$ comes from the calculations of

$$
M N^{-1}=\left[\begin{array}{ccc}
1 & 0 & 0 \\
\frac{2 u}{3} & \frac{(3+4 u)-\sqrt{9+12 u}}{6} & \frac{(3+4 u)+\sqrt{9+12 u}}{6} \\
\frac{2 u}{3} & \frac{(3+4 u)+\sqrt{9+12 u}}{6} & \frac{(3+4 u)-\sqrt{9+12 u}}{6}
\end{array}\right]
$$

and

$$
N M^{-1}=\left[\begin{array}{ccc}
1 & 0 & 0 \\
\frac{-2 u}{3+4 u} & \frac{\sqrt{3}(\sqrt{9+12 u}-(3+4 u))}{2(3+4 u) \sqrt{3+4 u}} & \frac{\sqrt{3}(\sqrt{9+12 u}+(3+4 u))}{2(3+4 u) \sqrt{3+4 u}} \\
\frac{-2 u}{3+4 u} & \frac{\sqrt{3}(\sqrt{9+12 u}+(3+4 u))}{2(3+4 u) \sqrt{3+4 u}} & \frac{\sqrt{3}(\sqrt{9+12 u}-(3+4 u))}{2(3+4 u) \sqrt{3+4 u}}
\end{array}\right] .
$$

The second rows of these matrices determine $\alpha, \beta, \gamma$ and $r, s, t$. Now we leave it to the reader to verify the formulas $\Delta\left(b_{0}\right)=b_{00}$,

$\Delta\left(b_{1}\right)=\frac{1}{4 u+3}\left(\left(4 u^{2}+2 u\right) b_{00}-2 u b_{01}+0 b_{02}-2 u b_{10}+(u+3) b_{11}-u b_{12}+0 b_{20}-u b_{21}+u b_{22}\right)$, 
and

$\Delta\left(b_{2}\right)=\frac{1}{4 u+3}\left(\left(4 u^{2}+2 u\right) b_{00}+0 b_{01}-2 u b_{02}+0 b_{10}+u b_{11}-u b_{12}-2 u b_{20}-u b_{21}+(u+3) b_{22}\right)$.

Example 6.4. Real Table Algebras of Dimension 3. Let $A$ be a real table algebra whose standard basis $\mathbf{B}=\left\{b_{0}, b_{1}, b_{2}\right\}$ satisfies $b_{i}^{*}=b_{i}$ for $i=0,1,2$. Let $\delta\left(b_{1}\right)=k$ and $\delta\left(b_{2}\right)=\ell$ be the degrees of the nonidentity basis elements, and let $u, v$ be the structure constants determined by $b_{1} b_{2}=u b_{1}+v b_{2}$. Since the degree map is an algebra homomorphism these parameters satisfy the necessary condition $k \ell=$ $k u+\ell v$. The remaining structure constants are determined by the identities

$$
b_{1}^{2}=k b_{0}+(k-u-1) b_{1}+(k-v) b_{2}, \text { and } b_{2}^{2}=\ell b_{0}+(\ell-u) b_{1}+(\ell-v-1) b_{2} .
$$

From the regular matrices of $b_{1}$ and $b_{2}$ we can determine the character table of $A$ relative to $\mathbf{B}$ in terms of the parameters $k, \ell, u$, and $v$.

\begin{tabular}{c|ccc|c} 
& 1 & $b_{1}$ & $b_{2}$ & $m_{\chi}$ \\
\hline$\delta$ & 1 & $k$ & $\ell$ & 1 \\
$\phi$ & 1 & $\frac{1}{2}((v-u-1)+\sqrt{\alpha})$ & $\frac{1}{2}((u-v-1)-\sqrt{\alpha})$ & $m_{\phi}$ \\
$\psi$ & 1 & $\frac{1}{2}((v-u-1)-\sqrt{\alpha})$ & $\frac{1}{2}((u-v-1)+\sqrt{\alpha})$ & $m_{\psi}$
\end{tabular}

where $\alpha=(v-u-1)^{2}+4 v$. The multiplicities can be calculated from the orthogonality relations, which lead to the equation $\frac{1+k+\ell}{m_{\phi}}=1+\frac{\phi\left(b_{1}\right)^{2}}{k}+\frac{\phi\left(b_{2}\right)^{2}}{\ell}$ and a similar one for $m_{\psi}$. These reduce to

$$
m_{\phi}=\frac{k \ell+k^{2} \ell+k \ell^{2}}{k \ell+\ell \phi_{1}^{2}+k \phi_{2}^{2}} \text { and } m_{\psi}=\frac{k \ell+k^{2} \ell+k \ell^{2}}{k \ell+\ell \psi_{1}^{2}+k \psi_{2}^{2}}
$$

where $\phi_{1}=\phi\left(b_{1}\right), \phi_{2}=\phi\left(b_{2}\right), \psi_{1}=\psi\left(b_{1}\right)$, and $\psi_{2}=\psi\left(b_{2}\right)$ are the corresponding character table entries.

Therefore, the change of basis matrix from the basis of primitive idempotents $E$ to $\mathbf{B}$ is

$$
M:=\left[\begin{array}{ccc}
1 & 1 & 1 \\
m_{\phi} & \frac{m_{\phi}}{k} \phi_{1} & \frac{m_{\phi}}{\ell} \phi_{2} \\
m_{\psi} & \frac{m_{\psi}}{k} \psi_{1} & \frac{m_{\psi}}{\ell} \psi_{2}
\end{array}\right]
$$

We know $A \simeq \mathbb{C} C_{3}$ as *-algebras. Let $N$ be the character table of $C_{3}$ given in the previous example. The change of basis matrix from the basis $C_{3}$ to our table 
algebra basis $\mathbf{B}$ will be

$$
\begin{aligned}
M N^{-1} & =\left[\begin{array}{ccc}
1 & 0 & 0 \\
\frac{m_{\phi}}{3}\left(1+\frac{\phi_{1}}{k}+\frac{\phi_{2}}{\ell}\right) & \frac{m_{\phi}}{3}\left(1+\frac{\phi_{1}}{k} \zeta^{2}+\frac{\phi_{2}}{\ell} \zeta\right) & \frac{m_{\phi}}{3}\left(1+\frac{\phi_{1}}{k} \zeta+\frac{\phi_{2}}{\ell} \zeta^{2}\right) \\
\frac{m_{\psi}}{3}\left(1+\frac{\psi_{1}}{k}+\frac{\psi_{2}}{\ell}\right) & \frac{m_{\psi}}{3}\left(1+\frac{\psi_{1}}{k} \zeta^{2}+\frac{\psi_{2}}{\ell} \zeta\right) & \frac{m_{\psi}}{3}\left(1+\frac{\psi_{1}}{k} \zeta+\frac{\psi_{2}}{\ell} \zeta^{2}\right)
\end{array}\right] \\
& :=\left[\begin{array}{ccc}
1 & 0 & 0 \\
\alpha_{1} & \beta_{1} & \bar{\beta}_{1} \\
\alpha_{2} & \beta_{2} & \bar{\beta}_{2}
\end{array}\right]
\end{aligned}
$$

and its inverse (obtained using Wolfram Math Alpha ${ }^{\circledR}$ ) is the change of basis matrix from $\mathbf{B}$ to $C_{3}$ :

$$
N M^{-1}=\left[\begin{array}{ccc}
1 & 0 & 0 \\
r & s & t \\
\bar{r} & \bar{s} & \bar{t}
\end{array}\right],
$$

where

$$
\begin{aligned}
r & =\frac{\phi_{1} \psi_{2}-\phi_{2} \psi_{1}+k\left(\phi_{2}-\psi_{2}\right) \zeta+\ell\left(\psi_{1}-\phi_{1}\right) \zeta^{2}}{\phi_{1}\left(\psi_{2}-\ell\right)+\phi_{2}\left(k-\psi_{1}\right)+\psi_{1} \ell-\psi_{2} k} \\
s & =\frac{\psi_{1} \ell-\psi_{2} k+k\left(\psi_{2}-\ell\right) \zeta+\ell\left(k-\psi_{1}\right) \zeta^{2}}{m_{\phi}\left(\phi_{1}\left(\psi_{2}-\ell\right)+\phi_{2}\left(k-\psi_{1}\right)+\psi_{1} \ell-\psi_{2} k\right)}, \text { and } \\
t & =\frac{\phi_{1} k-\phi_{2} \ell+k\left(\ell-\phi_{2}\right) \zeta+\ell\left(\phi_{1}-k\right) \zeta^{2}}{m_{\psi}\left(\phi_{1}\left(\psi_{2}-\ell\right)+\phi_{2}\left(k-\psi_{1}\right)+\psi_{1} \ell-\psi_{2} k\right)} .
\end{aligned}
$$

As before we can use $M N^{-1}$ and its inverse to derive a formula for the Hopf algebra comultiplication that is induced by the Hopf algebra comultiplication on the group algebra. We have that $b_{1}=\alpha_{1} b_{0}+\beta_{1} g+\bar{\beta}_{1} g^{-1}, b_{2}=\alpha_{2} b_{0}+\beta_{2} g+\bar{\beta}_{2} g^{-1}$, $g=r b_{0}+s b_{1}+t b_{2}$, and $g^{-1}=\bar{r} b_{0}+\bar{s} b_{1}+\bar{t} b_{2}$. Note that $\alpha_{1}$ and $\alpha_{2}$ are real follows from $b_{1}$ and $b_{2}$ being $*$-invariant. Again the ordering of the last two idempotents in the basis $E$ is not fixed, but switching the order only interchanges the second and third rows of these change of basis matrices. We will have

$$
\begin{aligned}
\Delta & \left(b_{1}\right)=\alpha_{1} \Delta\left(b_{0}\right)+\beta_{1} \Delta(g)+\bar{\beta}_{1} \Delta\left(g^{-1}\right) \\
= & \alpha_{1} b_{00}+\beta_{1}(g \otimes g)+\bar{\beta}_{1}\left(g^{-1} \otimes g^{-1}\right) \\
= & \alpha_{1} b_{00}+\beta_{1}\left[\left(r b_{0}+s b_{1}+t b_{2}\right) \otimes\left(r b_{0}+s b_{1}+t b_{2}\right)\right] \\
& \quad+\bar{\beta}_{1}\left[\left(\bar{r} b_{0}+\bar{s} b_{1}+\bar{t} b_{2}\right) \otimes\left(\bar{r} b_{0}+\bar{s} b_{1}+\bar{t} b_{2}\right)\right] \\
= & \left(\alpha_{1}+\beta_{1} r^{2}+\bar{\beta}_{1} \bar{r}^{2}\right) b_{00}+\left(\beta_{1} r s+\bar{\beta}_{1} \bar{r} \bar{s}\right) b_{01}+\left(\beta_{1} r t+\bar{\beta}_{1} \bar{r} \bar{t}\right) b_{02} \\
& +\left(\beta_{1} s r+\bar{\beta}_{1} \bar{s} \bar{r}\right) b_{10}+\left(\beta_{1} s^{2}+\bar{\beta}_{1} \bar{s}^{2}\right) b_{11}+\left(\beta_{1} s t+\bar{\beta}_{1} \bar{s} \bar{t}\right) b_{12} \\
& +\left(\beta_{1} t r+\bar{\beta}_{1} \bar{t} \bar{r}\right) b_{20}+\left(\beta_{1} t s+\bar{\beta}_{1} \bar{t} \bar{s}\right) b_{21}+\left(\beta_{1} t^{2}+\bar{\beta}_{1} \bar{t}^{2}\right) b_{22},
\end{aligned}
$$


and the formula for $\Delta\left(b_{2}\right)$ has the same pattern with $\alpha_{2}$ and $\beta_{2}$ replacing $\alpha_{1}$ and $\beta_{1}$. Note that the coefficients of the $b_{i j}$ occurring in $\Delta\left(b_{1}\right)$ and $\Delta\left(b_{2}\right)$ are guaranteed to be real numbers.

The explicit formulas in the 3-dimensional table algebra case are too long to express concisely here, but become easier in specific cases. We will demonstrate this with some actual calculations for table algebras corresponding to familiar stronglyregular graphs.

Example 6.5. The Hopf algebra structure of a rectangle. A rectangle is a stronglyregular graph on 4 vertices, which means that it generates an association scheme whose adjacency matrices form a table algebra basis. If $b_{2}$ is the $4 \times 4$ adjacency matrix of the rectangle, then the parameters of Example 6.4 for this table algebra are $k=1, \ell=2, u=0$, and $v=1$. Therefore, the character table of $\mathbb{C B}$ is

\begin{tabular}{c|ccc|c} 
& 1 & $b_{1}$ & $b_{2}$ & $m_{\chi}$ \\
\hline$\delta$ & 1 & 1 & 2 & 1 \\
$\phi$ & 1 & 1 & -2 & 1 \\
$\psi$ & 1 & -1 & 0 & 2
\end{tabular}

and the change of basis matrix from the group basis $C_{3}$ to the table algebra basis $\left\{b_{0}, b_{1}, b_{2}\right\}$ is

$$
M N^{-1}=\left[\begin{array}{ccc}
1 & 1 & 1 \\
1 & 1 & -1 \\
2 & -2 & 0
\end{array}\right] \cdot \frac{1}{3}\left[\begin{array}{ccc}
1 & 1 & 1 \\
1 & \zeta^{2} & \zeta \\
1 & \zeta & \zeta^{2}
\end{array}\right]=\left[\begin{array}{ccc}
1 & 0 & 0 \\
\frac{1}{3} & \frac{-2}{3} \zeta & \frac{-2}{3} \zeta^{2} \\
0 & -\frac{2}{3} \zeta-\frac{4}{3} \zeta^{2} & -\frac{4}{3} \zeta-\frac{2}{3} \zeta^{2}
\end{array}\right]
$$

Using this matrix and its inverse, we have $b_{1}=\frac{1}{3} e-\frac{2}{3} \zeta g-\frac{2}{3} \zeta^{2}, \quad b_{2}=\left(-\frac{2}{3} \zeta-\right.$ $\left.\frac{4}{3} \zeta^{2}\right) g+\left(-\frac{4}{3} \zeta-\frac{2}{3} \zeta^{2}\right) g^{2}$ and $g=\frac{1}{4} \zeta^{2} b_{0}-\frac{3}{4} \zeta^{2} b_{1}-\frac{1}{4}\left(2 \zeta+\zeta^{2}\right) b_{2}$, and $g^{2}=\frac{1}{4} \zeta b_{0}-$ $\frac{3}{4} \zeta b_{1}-\frac{1}{4}\left(\zeta+2 \zeta^{2}\right) b_{2}$. From these we can obtain formulas for $\Delta\left(b_{1}\right)$ and $\Delta\left(b_{2}\right)$ as before:

$$
\Delta\left(b_{1}\right)=\frac{1}{8}\left(3 b_{00}-b_{01}-b_{02}-b_{10}+3 b_{11}+3 b_{12}-b_{20}+3 b_{21}-b_{22}\right)
$$

and

$$
\Delta\left(b_{2}\right)=\frac{1}{8}\left(-b_{00}+3 b_{01}-b_{02}+3 b_{10}-9 b_{11}+3 b_{12}-b_{20}+3 b_{21}+3 b_{22}\right) .
$$

Example 6.6. The Hopf algebra structure of a pentagon. A pentagon is a strongly regular graph on 5 vertices whose adjacency matrix generates the real table algebra 
of dimension 3 with parameters $k=\ell=2$ and $u=v=1$. Its character table is

\begin{tabular}{c|ccc|c} 
& 1 & $b_{1}$ & $b_{2}$ & $m_{\chi}$ \\
\hline$\delta$ & 1 & 2 & 2 & 1 \\
$\phi$ & 1 & $\frac{-1+\sqrt{5}}{2}$ & $\frac{-1-\sqrt{5}}{2}$ & 2 \\
$\psi$ & 1 & $\frac{-1-\sqrt{5}}{2}$ & $\frac{-1+\sqrt{5}}{2}$ & 2
\end{tabular}

Therefore, the change of basis matrices between the bases $C_{3}$ and $\mathbf{B}$ are

$$
M N^{-1}=\left[\begin{array}{ccc}
1 & 0 & 0 \\
\frac{1}{3} & \frac{5+\sqrt{-15}}{6} & \frac{5-\sqrt{-15}}{6} \\
\frac{1}{3} & \frac{5-\sqrt{-15}}{6} & \frac{5+\sqrt{-15}}{6}
\end{array}\right] \text { and } N M^{-1}=\left[\begin{array}{ccc}
1 & 0 & 0 \\
\frac{-1}{5} & \frac{3-\sqrt{-15}}{10} & \frac{3+\sqrt{-15}}{10} \\
\frac{-1}{5} & \frac{3+\sqrt{-15}}{10} & \frac{3-\sqrt{-15}}{10}
\end{array}\right] .
$$

So the unique Hopf algebra structure on the adjacency algebra of a pentagon has its comultiplication given by

$$
\Delta\left(b_{1}\right)=\frac{1}{5}\left(2 b_{00}-b_{01}+0 b_{02}-b_{10}+b_{11}+2 b_{12}+0 b_{20}+2 b_{21}-2 b_{22}\right)
$$

and

$$
\Delta\left(b_{2}\right)=\frac{1}{5}\left(2 b_{00}+0 b_{01}-b_{02}+0 b_{10}-2 b_{11}+2 b_{12}-b_{20}+2 b_{21}+b_{22}\right) .
$$

Example 6.7. The Petersen graph is a strongly regular graph whose adjacency matrix generate the real table algebra of dimension 3 with parameters $k=3, \ell=6$, $u=2$, and $v=2$. Substituting these into our formulas, the character table of this table algebra is

\begin{tabular}{c|ccc|c} 
& 1 & $b_{1}$ & $b_{2}$ & $m_{\chi}$ \\
\hline$\delta$ & 1 & 3 & 6 & 1 \\
$\phi$ & 1 & -2 & 1 & 4 \\
$\psi$ & 1 & 1 & -2 & 5
\end{tabular}

so our change of basis matrix is

$$
M=\left[\begin{array}{ccc}
1 & 1 & 1 \\
4 & -\frac{8}{3} & \frac{2}{3} \\
5 & \frac{5}{3} & -\frac{5}{3}
\end{array}\right]
$$

From this matrix and the character table $N$ of the group of order 3 we can produce our formula for the Hopf algebra comultiplication on the adjacency algebra of the Petersen graph:

$$
\Delta\left(b_{1}\right)=\frac{1}{10}\left(5 b_{00}-5 b_{01}+3 b_{02}-5 b_{10}+0 b_{11}+3 b_{12}+3 b_{20}+3 b_{21}-3 b_{22}\right)
$$

and

$$
\Delta\left(b_{2}\right)=\frac{1}{10}\left(22 b_{00}-3 b_{01}-2 b_{02}-3 b_{10}-3 b_{11}+3 b_{12}-2 b_{20}+3 b_{21}+0 b_{22}\right) .
$$


Acknowledgement. The authors would like to express their gratitude to the referee for many helpful comments, and in particular for suggesting a better proof of Theorem 5.1.

\section{References}

[1] H. I. Blau, Table algebras, European J. Combin., 30(6) (2009), 1426-1455.

[2] A. Hanaki, Character products of association schemes, J. Algebra, 283(2) (2005), 596-603.

[3] A. Herman, M. Muzychuk and B. Xu, The recognition problem for table algebras and reality-based algebras, J. Algebra, 479 (2017), 173-191.

[4] A. Masuoka, Semisimple Hopf algebras of dimension 6,8, Israel J. Math., 92 (1995), 361-373.

[5] A. Masuoka, The $p^{n}$ theorem for semisimple Hopf algebras, Proc. Amer. Math. Soc., 124 (1996), 735-737.

[6] D. E. Radford, Hopf Algebras, Series on Knots and Everything, 49, World Scientific Publishing Co. Pte. Ltd., Hackensack, NJ, 2012.

[7] Y. Zhu, Hopf algebras of prime dimension, Internat. Math. Res. Notices, 1 (1994), 53-59.

\footnotetext{
Allen Herman (Corresponding Author) and Gurmail Singh

Department of Mathematics and Statistics

University of Regina

Regina, Canada, S4S 0A2

e-mails: Allen.Herman@uregina.ca (A. Herman)

singh28g@uregina.ca (G. Singh)
} 